\title{
The role of novel biomarkers in childhood idiopathic nephrotic syndrome: a narrative review of published evidence
}

This article was published in the following Dove Press journal: International Journal of Nephrology and Renovascular Disease I June 2017

Number of times this article has been viewed

\section{Samuel N Uwaezuoke \\ Department of Pediatrics, Pediatric Nephrology Firm, University of Nigeria Teaching Hospital, Ituku- Ozalla, Enugu, Nigeria}

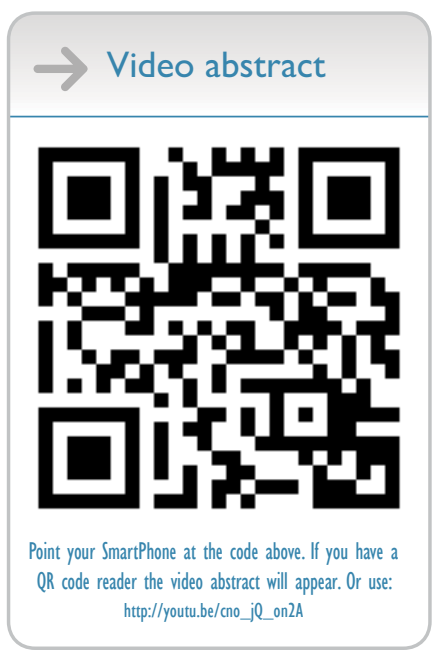

Correspondence: Samuel N Uwaezuoke Department of Pediatrics, UNTH, PMB 0I449, Enugu 40000I, Nigeria Tel +2348033248108 Email snuwaezuoke@yahoo.com

\begin{abstract}
Two histological subtypes of idiopathic nephrotic syndrome are commonly recognized in children, namely minimal change nephropathy and focal segmental glomerulosclerosis. Children with minimal change nephropathy (the majority of whom are steroid-sensitive) and focal segmental glomerulosclerosis (the majority of whom are steroid-resistant) require early identification in order to ensure appropriate therapeutic intervention and better outcome. Although renal biopsy and histology remain the ideal diagnostic steps to identify these histological subtypes, reports indicate that serum and urinary biomarkers are now being utilized in the investigation of childhood idiopathic nephrotic syndrome. This paper aims to review the diagnostic and prognostic utility of novel biomarkers in childhood idiopathic nephrotic syndrome and to highlight their role in differentiating steroid-sensitive nephrotic syndrome (SRNS) from steroid-resistant nephrotic syndrome (SSNS). Using the terms "idiopathic nephrotic syndrome," "children," and "biomarkers" the PubMed database was searched for relevant studies related to the topic. Biomarkers such as adiponectin, neopterin, $\beta 2$-microglobulin, and N-acetyl- $\beta$-D glucosaminidase were reported as diagnostic markers. In addition to neopterin and $\mathrm{N}$-acetyl$\beta$-D glucosaminidase, urine vitamin D-binding protein and $\alpha 1 \beta$-glycoprotein were shown to differentiate SRNS from SSNS while N-acetyl- $\beta$-D glucosaminidase and $\beta 2$-microglobulin could predict steroid responsiveness and renal outcome in SRNS. Although progress has been made in demonstrating the diagnostic and prognostic utility of these biomarkers, their limited availability in most laboratories has precluded a complete paradigm shift from the conventional renal biopsy. Nevertheless, further longitudinal studies are required to establish their usefulness as noninvasive predictors of disease response to immunosuppressive therapy.
\end{abstract}

Keywords: biomarkers, idiopathic nephrotic syndrome, children, investigation

\section{Introduction}

Nephrotic syndrome is the most common presentation of glomerular disease in children. ${ }^{1}$ The syndrome is characterized by the tetrad of massive proteinuria (more than $1 \mathrm{~g} / \mathrm{m}^{2} / 24 \mathrm{~h}$ ), hypoalbuminemia, generalized edema, and hyperlipidemia. It can be congenital or acquired; the acquired causes can either be idiopathic (primary) or secondary. Two histological subtypes of idiopathic nephrotic syndrome are commonly recognized in children, namely, minimal change nephropathy (MCN) and focal segmental glomerulosclerosis (FSGS). ${ }^{1}$

An earlier report by the International Study of Kidney Disease in Children suggests that a preponderance of preadolescent children with idiopathic nephrotic syndrome presents with $\mathrm{MCN}$ on renal biopsy. ${ }^{2}$ More than $90 \%$ of children with this histological subtype achieve remission with oral corticosteroids; hence, MCN is also identified as 
steroid-sensitive nephrotic syndrome (SSNS). ${ }^{3}$ However, frequent relapses and steroid dependence are disease outcomes in about $20 \%-60 \%$ of affected children. ${ }^{4}$ Conversely, FSGS is characterized by steroid resistance in a majority of patients, steroid sensitivity in only about $20 \%$ of cases, as well as a high risk of developing end-stage renal disease. ${ }^{5}$

Various hypotheses regarding the pathogenesis of the syndrome have evolved over time, with the current paradigm revolving around a podocytopathy. ${ }^{6,7}$ The podocyte clearly plays a critical role in maintaining the glomerular filtration barrier and structural integrity; thus, podocyte injury and loss contribute to proteinuria and progressive sclerosis. ${ }^{8}$ For instance, one report indicates that the podocyte expresses receptors for interleukin-4 and interleukin-13, which, if activated by these cytokines, might disrupt glomerular permeability resulting in proteinuria. ${ }^{9}$ Podocyte injury can occur in several immunologic and nonimmunologic diseases of the kidney. Idiopathic MCN and FSGS (acquired podocytopathies) are considered as immunologic diseases. Notably, immunosuppressive agents such as corticosteroids and calcineurin inhibitors are known to protect the podocyte against injury and loss by stabilizing its actin cytoskeleton, cell maturation, and survival: an action that underscores their anti-proteinuric effect in idiopathic nephrotic syndrome. ${ }^{8}$ In fact, the use of calcineurin inhibitors as the initial therapy for children with steroid-resistant nephrotic syndrome (SRNS) is the current evidence-based recommendation in the treatment guideline for glomerulonephritis. ${ }^{10}$

Idiopathic MCN (SSNS) and FSGS (SRNS) require early differentiation in order to ensure prompt therapeutic intervention and better outcome. For instance, Jiang and Luo observed that SRNS was associated with elevated serum interleukin-18 (IL-18) level and IL-18 micro RNA (mRNA) expression in peripheral blood mononuclear cells of children with primary or idiopathic nephrotic syndrome, suggesting that overproduction of IL-18 may be relevant in the development of SRNS. ${ }^{11}$ Interestingly, several other reports have also shown that serum and urinary biomarkers are useful in the diagnostic evaluation of childhood idiopathic nephrotic syndrome. ${ }^{12-20}$ In one of these studies, Bennett et al $^{14}$ recently documented in a preliminary report that urinary vitamin D-binding protein (uVDBP) represents a novel biomarker that could significantly differentiate SRNS from SSNS. While some of the biomarkers have a poor diagnostic utility, ${ }^{17}$ others have a high discriminatory power for differentiating SRNS from SSNS, apart from serving as reliable predictors of steroid responsiveness. ${ }^{14,16-20}$
This paper aims to review the diagnostic and prognostic utility of novel biomarkers in childhood idiopathic nephrotic syndrome and to highlight their role in the differentiation of SRNS from SSNS.

\section{Literature search}

Using these terms "idiopathic nephrotic syndrome," "children," and "biomarkers" the PubMed database was searched for relevant studies related to the topic. Studies included for review were conducted in children and published not more than 20 years ago. Information was also gathered from a few other reports irrespective of the year of publication.

\section{The diagnostic, prognostic, and discriminatory roles of biomarkers}

With respect to biomarkers of renal injury, an ideal example should have the following characteristics: its secretion after cell injury and its organ specificity, its level being proportionate to the extent of cell injury, its early production after a reversible organ injury, its quick reduction in level after injury to ensure its use as a treatment-monitoring tool, and its ability to be promptly and reliably estimated. ${ }^{21}$ In fact, proteomic biomarkers constitute a potential armamentarium for enhancing the management of patients with renal diseases by enabling a more accurate and earlier detection. ${ }^{22}$ Several novel biomarkers have recently been documented as diagnostic and prognostic tools in diseases such as pediatric acute kidney injury, ${ }^{23-36}$ and urinary tract infection. ${ }^{37-43}$

Similarly, the utility of biomarkers in childhood idiopathic nephrotic syndrome has been reported; ${ }^{12-20}$ their application constitutes a noninvasive approach in diagnostic nephrology, as they can be used as diagnostic and prognostic tools in idiopathic nephrotic syndrome, as well as discriminatory tools in distinguishing SRNS from SSNS (Table 1).

For example, Bakr et $\mathrm{al}^{13}$ have reported the diagnostic utility of serum neopterin levels in children with idiopathic or primary nephrotic syndrome. In their study, the researchers investigated two groups of children with the disease: 38 patients with active idiopathic nephrotic syndrome (comprising 28 SSNS patients and 10 SRSN patients) (group I) and 17 patients with idiopathic nephrotic syndrome who were in remission (group II), as well as 20 healthy controls. Serum neopterin levels were estimated in the cohorts, and all patients in these cohorts had normal creatinine clearance. Interestingly, the serum neopterin levels were significantly elevated among the group I patients with the active disease in comparison to group II patients in remission, and even the controls. Notably, in patients in remission and the controls, 
Table I Studies reporting the role of novel biomarkers in childhood idiopathic nephrotic syndrome

\begin{tabular}{|c|c|c|c|c|}
\hline Study & Country & Study method & $\begin{array}{l}\text { Novel biomarkers } \\
\text { (body fluid) }\end{array}$ & $\begin{array}{l}\text { Reported role of } \\
\text { biomarkers }\end{array}$ \\
\hline Bakkalŏglu et al, ${ }^{12} 2005$ & Turkey & Cross-sectional, case-control & Adiponectin (serum) & Diagnostic $^{\mathrm{a}}$ \\
\hline \multirow[t]{2}{*}{ 'Bakr et al, ${ }^{13} 2006$} & Egypt & Cross-sectional, case-control & Neopterin (serum) & Diagnostic $^{b}$ \\
\hline & & & & Discriminatory $^{c}$ \\
\hline Bennett et al, ${ }^{14} 2016$ & USA & Cross-sectional, case-control & Vitamin D binding protein (urine) & Discriminatory ${ }^{c}$ \\
\hline \multirow[t]{2}{*}{ Mishra et al, ${ }^{16} 2012$} & India & Cross-sectional, case-control & NAG (urine) & Discriminatory $^{c}$ \\
\hline & & & & Prognostic $^{d}$ \\
\hline Piyaphanee et al, ${ }^{18} 201 \mathrm{I}$ & USA & Cross-sectional, case-control & AIBG (I3.8 kDa fragment) (urine) & Discriminatory $^{c}$ \\
\hline Fede et al, ${ }^{19} 1999$ & Italy & Cross-sectional, case-control & $\beta 2 M$ NAG (urine) & Prognostic \\
\hline Calișkan et al, ${ }^{46} 1996$ & Turkey & Cross-sectional & $\beta 2 M$ NAG (urine) & Diagnostic ${ }^{f}$ \\
\hline
\end{tabular}

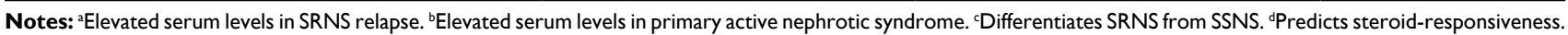
ePredicts tubular injury and dysfunction in SRNS. 'Elevated levels in SRNS and SSNS.

Abbreviations: $\beta 2 \mathrm{M}, \beta 2$-microglobulin; AIBG, $\alpha$ I- $\beta$ glycoprotein; NAG, N-acetyl- $\beta$-D glucosaminidase; SRNS, steroid-resistant nephrotic syndrome; SSNS, steroidsensitive nephrotic syndrome.

similar neopterin levels were observed. While a significant positive correlation between the levels of the biomarker and the degree of proteinuria was observed in group I patients, no significant differences in its levels were noted between SSNS and SRNS patients. ${ }^{13}$ The findings of these authors highlighted the diagnostic utility of neopterin for active idiopathic nephrotic syndrome, but underscored its poor discriminatory ability for SSNS and SRNS.

Other investigators have reported the role of adipokines in the investigation of idiopathic nephrotic syndrome; ${ }^{12,44}$ these adipokines - adiponectin (ADPN), complement 3 (C3), and acylation stimulating protein (ASP) - whose plasma levels are altered in childhood obesity in the absence of lipid changes may specifically predispose to enhanced fat storage(mediated by ASP) and decreased fat oxidation (mediated by ADPN). ${ }^{45}$

Thus, Bakkalŏglu et a ${ }^{12}$ evaluated the effect of nephrotic state on serum ADPN levels in pediatric patients with SRNS by comparing the levels in relapse and remission as well as in controls, and they noted that elevated serum ADPN levels were found in SRNS patients in relapse compared to those in remission. For instance, strong positive correlations were observed between serum ADPN levels and lipid parameters/ proteinuria. Conversely, negative correlations were documented between ADPN levels and serum protein/albumin levels. This observation possibly reflects a compensatory response to the nephrotic state which is characterized by massive proteinuria, hypoalbuminemia, and hyperlipidemia. Other researchers in China sought to examine the relationship between serum ASP level/serum C3 level and blood lipids in children with idiopathic nephrotic syndrome by estimating the fasting serum ASP, C3, albumin, and blood lipids of the patients with proteinuria, those in remission, and their healthy counterparts who served as controls. ${ }^{44}$ Unlike serum $\mathrm{C} 3$ levels, the serum ASP levels in the proteinuria group were observed to be significantly higher than those in the remission and the control groups. Furthermore, the serum levels of total cholesterol, triglyceride, low-density lipoprotein, and apolipoprotein B were elevated in the proteinuria group when compared with the remission and the control groups. Serum ASP levels were positively correlated with serum C3 and triglyceride levels, but serum C3 levels were not correlated with serum triglyceride levels: underscoring the fact that the elevated serum ASP level may be linked with a complement-related mechanism against hyperlipidemia in children with the disease. ${ }^{44}$

In a recent cross-sectional study, Bennett et al ${ }^{14}$ reported that uVDBP could distinguish SRNS from SSNS with a high discriminatory power; the researchers tested the hypothesis that the biomarker represents a discriminatory tool for both forms of nephrotic syndrome, given its increased urinary excretion in idiopathic nephrotic syndrome. Remarkably, they observed that levels of uVDBP were significantly higher in patients with SRNS than in patients with SSNS and normal controls: a finding that was unchanged even after correction for urine creatinine. There was a significant negative correlation between $\mathrm{UVDBP}$ and estimated glomerular filtration rate, although uVDBP remained markedly increased in SRNS patients with normal estimated glomerular filtration rate. Despite the positive correlation between microalbuminuria and UVDB, the latter showed a higher discriminatory ability for differentiating SRNS from SSNS than the former. ${ }^{14}$

Mishra et $a{ }^{16}$ evaluated the differential excretion of urinary N-acetyl- $\beta$-D glucosaminidase (NAG) among three subgroups of patients with idiopathic nephrotic syndrome, namely, those who presented with first episode (FENS patients), those with relapses (RNS patients), and those with steroid resistance (SRNS patients), as the enzyme is 
a sensitive biomarker of renal parenchymal disease. The enrolled patients were investigated alongside age-and sexmatched normal children who served as controls. The major findings of their study were the significant elevation in urinary NAG/creatinine levels among SRNS patients as compared to FENS and RNS patients, similar biomarker levels among the FENS and RNS patients, significant correlations between experimental and predicted values of urinary NAG/creatinine in SSNS and SRNS, and observation of higher values in SRNS than in SSNS patients, since it has moderate predictive value for steroid sensitivity or responsiveness. ${ }^{16}$ Thus, this urine biomarker obviously has both prognostic and discriminatory roles in childhood idiopathic nephrotic syndrome.

In a study in South Korea, another biomarker (urinary exosomal WT1) - recently suggested as a novel biomarker for podocytopathy - was investigated by Lee et a ${ }^{17}$ in order to determine its role in predicting steroid sensitivity or renal pathological state in patients with idiopathic nephrotic syndrome. Although the biomarker was detectable in $62.5 \%$ of the patients they studied, there was no significant difference in the degree of proteinuria between patients with or without the biomarker and no significant difference in the number of the patients with the detectable biomarker according to steroid sensitivity or renal pathological state - findings which failed to verify its role as a reliable biomarker in childhood idiopathic nephrotic syndrome. ${ }^{17}$

In a cross-sectional pilot study, another group of researchers also evaluated a novel biomarker that could potentially differentiate SRNS from SSNS; ${ }^{18}$ urine and clinical data were gathered from pediatric patients with idiopathic nephrotic syndrome, as well as from healthy controls. Interestingly, this biomarker - the $13.8 \mathrm{kDa}$ fragment of $\alpha 1-\beta$ glycoprotein - was detected in urine in about $37 \%$ of the patients with SRNS who also had lower GFR, but was not detected in their counterparts with SSNS and the controls. ${ }^{18}$ In this study, the biomarker clearly showed a high discriminatory ability for steroid resistance in nephrotic syndrome, albeit present in only a subset of patients.

To corroborate the report of Mishra et al, ${ }^{16}$ Fede et al ${ }^{19}$ assessed urinary excretion of NAG with $\beta 2$-microglobulin $(\beta 2 \mathrm{M})$ in children with nephrotic syndrome as increased urinary excretion of both biomarkers is considered an indicator of tubulo-interstitial disease. They studied three subgroups of children: those with SSNS, those with SRNS, and ageand sex-matched healthy controls. In summary, the authors found that at onset, excretion of NAG was significantly higher in children with SSNS than in controls, whereas in SRNS at onset, excretion of NAG and $\beta 2 \mathrm{M}$ was significantly higher than in SSNS and remained the same at the end of steroid therapy; the findings suggest that urinary excretion of NAG and $\beta 2 \mathrm{M}$ may be a reliable indicator of the tubular dysfunction that is usually seen in SRNS patients. ${ }^{19}$ Generally, enzymuria reflects tubular injury, while low-molecularweight proteinuria points to tubular dysfunction. In the study by Calişkan et al, ${ }^{46}$ increased excretion of urinary NAG and $\beta 2 \mathrm{M}$ were clearly demonstrated in pediatric patients with SSNS and SRNS. There was a positive correlation between proteinuria and urinary $\mathrm{NAG}$ and $\beta 2 \mathrm{M}$ excretion in these patients indicating that heavy glomerular proteinuria may lead to a marked urinary NAG excretion and a moderate urinary $\beta 2 \mathrm{M}$ elevation independent of primary renal pathology. Other investigators who compared the accuracy of urinary $\beta 2 \mathrm{M}$ and NAG in predicting renal insufficiency and remission rates in adult patients with idiopathic membranous nephropathy, however, reported that $\beta 2 \mathrm{M}$ was more superior than NAG as a prognostic tool. ${ }^{47}$ Even though both biomarkers were found to be predictors of renal outcome in idiopathic membranous nephropathy, $\beta 2 \mathrm{M}$ was noted to be more accurate in this prognostic role.

Furthermore, the prognostic utility of $\beta 2 \mathrm{M}$ in patients with this form of nephropathy has also been reported by Reichert et al. ${ }^{48}$ In a longitudinal study, the authors estimated urinary $\beta 2 \mathrm{M}$ in 30 adult patients with the nephropathy, a nephrotic syndrome, and normal renal function. Remarkably, $\beta 2 \mathrm{M}$ excretion correlated with deterioration of renal function: suggesting that its measurement may not only help in identifying patients at high risk for renal insufficiency but may also serve as a guide for early immunosuppressive therapy. ${ }^{48}$ Their findings are in agreement with the report of Branten et $\mathrm{al}^{49}$ who noted that urinary immunoglobulin $\mathrm{G}$ and $\beta 2 \mathrm{M}$ are reliable predictors of renal insufficiency in idiopathic membranous nephropathy. Their prospective study, which was similarly conducted on adult patients, revealed that urinary $\beta 2 \mathrm{M}$ was the strongest independent predictor of adverse renal outcome. However, combining the excretions of both macromolecules significantly improved the specificity for predicting renal insufficiency in these patients with primary nephropathy.

Interestingly, the prognostic utility of NAG was further elucidated by Bazzi et $\mathrm{al}^{50}$ in another study of adult 136 patients with primary glomerulonephritis; the authors observed that urinary NAG excretion values were significantly correlated with 24-hour proteinuria, IgG excretion, and fractional excretion (FE) of $\alpha(1)$ microglobulin $(\alpha(1)$ $m)$. The finding underscores the fact that urinary NAG excretion appears to be a dependable marker of proteinuria- 
related tubule toxicity in the early stage of the histological subtypes of primary glomerulonephritis, namely, idiopathic membranous nephropathy, FSGS, and $\mathrm{MCN} .{ }^{50}$ The estimation of this biomarker could also serve as a useful parameter for prompt identification of patients who would later develop chronic renal failure or go into clinical remission or achieve response to immunosuppressive treatment.

Given the inability of any clinical or histological feature to predict responsiveness to therapy, another group of researchers assessed whether FE of IgG (FE IgG) and $\alpha$ (1)m correlated with histological lesions; whether these markers could predict outcome; and whether they could serve as therapeutic guides. ${ }^{51}$ In the study of 50 patients with FSGS, the authors demonstrated that FE IgG showed the best predictive value for remission, progression, and response to therapy, and hence may be utilized to guide therapy. Specifically, it was noted that in these patients, FE IgG was at the limit of statistically significant relationship with segmental sclerosis, while FE $\alpha$ (1)m was associated with tubulo-interstitial injury. ${ }^{51}$

\section{Conclusion}

Biomarkers are useful tools for diagnosis and prognostication in both childhood and adult idiopathic nephrotic syndrome, as well as for differentiating SRNS from SSNS. Currently, clinical and histological features are still dependable guides for predicting disease responsiveness to steroid therapy. However, biomarkers can now be utilized to predict renal outcome in the disease, as well as to promptly identify those patients who would respond to immunosuppressive therapy. Although progress has been made in demonstrating the prognostic and discriminatory roles of these biomarkers, their limited availability in most laboratories has precluded a complete paradigm shift from the conventional renal biopsy. Nevertheless, further longitudinal studies are still required to establish their usefulness as noninvasive predictors of disease response to immunosuppressive therapy.

\section{Disclosure}

The author reports no conflicts of interest in this work.

\section{References}

1. Eddy AA, Symons JM. Nephrotic syndrome in childhood. Lancet. 2003;362:629-639.

2. Nephrotic syndrome in children: prediction of histopathology from clinical and laboratory characteristics at time of diagnosis. A report of the International Study of Kidney Disease in Children. Kidney Int. 1978;13:159-165.

3. Trumpeter RS, Lloyd BW, Hicks J, White RH, Cameron JS. Long-term outcome for children with minimal change nephrotic syndrome. Lancet. $1985 ; 1: 368-370$
4. Teeninga N, Kist-van Holthe JE, Nauta J. Extending prednisolone treatment does not reduce relapses in childhood nephrotic syndrome. J Am Soc Nephrol. 2012;24:149-159.

5. Primary nephrotic syndrome in children: clinical significance of histopathologic variants of minimal change and of diffuse mesangial hypercellularity. A report of the International Study of Kidney Disease in Children. Kidney Int. 1981;20:765-771.

6. Uwaezuoke SN. Steroid-sensitive nephrotic syndrome in children: triggers of relapse and evolving hypotheses on pathogenesis. Italian $J$ Pediatr. 2015;41:19.

7. Greenbaum LA, Benndorf R, Smoyer WE. Childhood nephrotic syndrome: current and future therapies. Nat Rev Nephrol. 2012;8:445-458.

8. Schonenberger E, Ehrich JH, Haller H, Schiffer M. The podocyte as a direct target of immunosuppressive agents. Nephrol Dial Transplant. 2011;26:18-24.

9. Van Den Berg JG, Aten J, Chand MA, et al. Interleukin-4 and interleukin-13 act on glomerular visceral epithelial cells. J Am Soc Nephrol. 2000;11:413-422.

10. Kidney Disease: Improving Global Outcomes (KDIGO) Glomerulonephritis Work Group. KDIGO Clinical Practice Guideline for Glomerulonephritis. Kidney Int. 2012;2:139-274.

11. Jiang HK, Luo G. Interleukin-18 expression in peripheral blood mononuclear cells in children with steroid-resistant nephrotic syndrome. Chinese J Contemp Pediatr. 2009;11(5):337-340.

12. Bakkalŏglu SA, Soylemezoglu O, Buyan N, et al. High serum adiponectin levels during steroid-responsive nephrotic syndrome relapse. Pediatr Nephrol. 2005;20(4):474-477.

13. Bakr A, Rageh I, el-Azouny M, Deyab S, Lotfy H. Serum neopterin levels in children with primary nephrotic syndrome. Acta Paediatr. 2006;95(7):854-856.

14. Bennett MR, Pordal A, Haffner C, Pleasant L, Ma Q, Devarajan P. Urinary vitamin D-binding protein as a biomarker of steroid-resistant nephrotic syndrome. Biomarker Insights. 2016;11:1-6.

15. Bai Y, Liu W, Guo Q, Zou Y. Screening for urinary biomarkers of steroid-resistant nephrotic syndrome in children. Exp Ther Med. 2013;5(3):860-864.

16. Mishra OP, Jain P, Srivastava P, Prasad R. Urinary N-acetyl-beta-D glucosaminidase (NAG) level in idiopathic nephrotic syndrome. Pediatr Nephrol. 2012;27(4):589-596.

17. Lee H, Han KH, Lee SE, Kim SH, Kang HG, Cheong HI. Urinary exosomal WT1 in childhood nephrotic syndrome. Pediatr Nephrol. 2012;27(2):317-320.

18. Piyaphanee N, Ma Q, Kremen O, et al. Discovery and initial validation of $\alpha 1$-B glycoprotein fragmentation as a differential urinary biomarker in pediatric steroid-resistant nephrotic syndrome. Proteomics Clin Appl. 2011;5(5-6):334-342.

19. Fede C, Conti G, Chimenz R, Ricca M. N-acetyl-beta-D-glucosaminidase and beta2-microglobulin: prognostic markers in idiopathic nephrotic syndrome. J Nephrol. 1999;12(1):51-55.

20. Traum AZ. Urine proteomic profiling to identify biomarkers of steroid resistance in pediatric nephrotic syndrome. Expert Rev Proteomics. 2008;5(5):715-719.

21. Cruz DN, Goh CY, Haase-Fielitz A, Ronco C, Haase M. Early biomarkers of renal injury. Congest Heart Fail. 2010;16:S25-S31.

22. Mischak H, Delles C, Vlahou A, Vanholder R. Proteomic biomarkers in kidney disease: issues in development and implementation. Nat Rev Nephrol. 2015;11:221-232.

23. Uwaezuoke SN. Acute kidney injury in children: enhancing diagnosis with novel biomarkers. J Acute Dis. 2016;5(4):267-270.

24. de Geus HR, Betjes MG, Bakker J. Biomarkers for the prediction of acute kidney injury: a narrative review on current status and future challenges. Clin Kidney J. 2012;5(2):102-108.

25. Shao X, Tian L, Xu W, et al. Diagnostic value of urinary kidney injury molecule 1 for acute kidney injury: a meta-analysis. PloS One. 2014;9(1):e84131. 
26. Schinstock CA, Semret MH, Wagner SJ, et al. Urinalysis is more specific and urinary neutrophil gelatinase-associated lipocalin is more sensitive for early detection of acute kidney injury. Nephrol Dial Transpl. 2013;28(5):1175-1185.

27. Yim HE. Neutrophil gelatinase-associated lipocalin and kidney diseases. Child Kidney Dis. 2015;19(2):79-88.

28. Bongiovanni C, Magrini L, Salerno G, et al. Serum cystatin C for the diagnosis of acute kidney injury in patients admitted in the emergency department. Dis Markers. 2015;2015:416059.

29. Park MY, Choi SJ, Kim JK, Hwang SD, Lee YW. Urinary cystatin C levels as a diagnostic and prognostic biomarker in patients with acute kidney injury. Nephrology (Carlton). 2013;18(4):256-262.

30. Treeprasertsuk S, Wongkarnjana A, Jaruvongvanich V, et al. Urine neutrophil gelatinase-associated lipocalin: a diagnostic and prognostic marker for acute kidney injury (AKI) in hospitalized cirrhotic patients with AKI-prone conditions. BMC Gastroenterol. 2015;15:140.

31. Barreto R, Elia C, Solà E, et al. Urinary neutrophil gelatinase-associated lipocalin predicts kidney outcome and death in patients with cirrhosis and bacterial infections. J Hepatol. 2014;61(1):35-42.

32. Zhang A, Cai Y, Wang PF, et al. Diagnosis and prognosis of neutrophil gelatinase-associated lipocalin for acute kidney injury with sepsis: a systematic review and meta-analysis. Crit Care. 2016;20:41.

33. Aregger F, Uehlinger DE, Witowski J, et al. Identification of IGFBP-7 by urinary proteomics as a novel prognostic marker in early acute kidney injury. Kidney Int. 2014;85(4):909-919.

34. Dewitte A, Joannès-Boyau $\mathrm{O}$, Sidobre $\mathrm{C}$, et al. Kinetic eGFR and novel AKI biomarkers to predict renal recovery. Clin J Am Soc Nephrol. 2015;10(11):1900-1910.

35. Alge JL, Karakala N, Neely BA, et al. Urinary angiotensinogen and risk of severe AKI. Clin J Am Soc Nephrol. 2013;8(2):184-193.

36. Westhoff JH, Tönshoff B, Waldherr S, et al. Urinary tissue inhibitor of metalloproteinase-2 (TIMP-2) • insulin-like growth factor-binding protein 7 (IGFBP7) predicts adverse outcome in pediatric acute kidney injury. PLoS One 2015;10(11):e0143628.

37. Yim HE, Yim H, Bae ES, Woo SU, Yoo KH. Predictive value of urinary and serum biomarkers in young children with febrile urinary tract infections. Pediatr Nephrol. 2014;29(11):2181-2189.

38. Leroy S, Gervaix A. Procalcitonin, a useful biomarker in pediatric urinary tract infection. Arch Pediatr. 2013;20(1):54-62.

39. Kim BH, Yu N, Kim HR, et al. Evaluation of the optimal neutrophil gelatinase-associated lipocalin value as a screening biomarker for urinary tract infections in children. Ann Lab Med. 2014;34(5):354-359.
40. Leroy S, Fernandez-Lopez A, Nikfar R, et al. Association of procalcitonin with acute pyelonephritis and renal scars in pediatric UTI. Pediatrics. 2013;131(5):870-879.

41. Nanda M, Juthani-Mehta M. Novel biomarkers for the diagnosis of urinary tract infection-a systematic review. Biomarker Insights. 2009;4:111-121.

42. Ghasemi K, Esteghamati M, Borzoo S, Parvaneh E, Borzoo S. Predictive accuracy of urinary neutrophil gelatinase associated lipocalin (NGAL) for renal parenchymal involvement in children with acute pyelonephritis. Electron Physician. 2016;8(2):1911-1917.

43. Chien JW, Wang LY, Cheng YS, Tsai YG, Liu CS. Urinary 8-hydroxy2'-deoxy guanosine (8-oxodG) level can predict acute renal damage in young children with urinary tract infection. Biomarkers. 2014;19(4):326-331.

44. Wang JJ, Huang Y, Wu C, Xu YX, Wei YS, Zhao Y. Levels of serum acylation-stimulating protein and $\mathrm{C} 3$ in children with primary nephrotic syndrome. Chinese J Contemp Pediatr. 2011;13(10):817-819.

45. Cianflone K, Lu H, Smith J, Yu W, Wang H. Adiponectin, acylation stimulating protein and complement $\mathrm{C} 3$ are altered in obesity in very young children. Clin Endocrinol. 2005;62(5):567-572.

46. Calişkan S, Hacibekiroğlu M, Sever L, Ozbay G, Arisoy N. Urinary $\mathrm{N}$-acetyl-beta-D-glucosaminidase and beta 2-microglobulin excretion in primary nephrotic children. Nephron. 1996;74(2):401-404.

47. Hofstra JM, Deegens JK, Willems HL, Wetzels JF. Beta-2-microglobulin is superior to $\mathrm{N}$-acetyl-beta-glucosaminidase in predicting prognosis in idiopathic membranous nephropathy. Nephrol Dial Transplant. 2008;23(8):2546-2551.

48. Reichert LJ, Koene RA, Wetzels JF. Urinary excretion of beta 2-microglobulin predicts renal outcome in patients with idiopathic membranous nephropathy. J Am Soc Nephrol. 1995;6:1666-1669.

49. Branten AJ, du Buf-Vereijken PW, Klasen IS, et al. Urinary excretion of beta2-microglobulin and IgG predict prognosis in idiopathic membranous nephropathy: a validation study. J Am Soc Nephrol. 2005;16:169-174.

50. Bazzi C, Petrini C, Rizza V, et al. Urinary N-acetyl-beta-glucosaminidase excretion is a marker of tubular cell dysfunction and a predictor of outcome in primary glomerulonephritis. Nephrol Dial Transplant. 2002;17(11):1890-1896.

51. Bazzi C, Petrini C, Rizza V, et al. Fractional excretion of IgG predicts renal outcome and response to therapy in primary focal segmental glomerulosclerosis: a pilot study. Am J Kidney Dis. 2003;41(2):328-335.

\section{Publish your work in this journal}

The International Journal of Nephrology and Renovascular Disease is an international, peer-reviewed open access journal focusing on the pathophysiology of the kidney and vascular supply. Epidemiology, screening, diagnosis, and treatment interventions are covered as well as basic science, biochemical and immunological studies. The manuscript management system is completely online and includes a very quick and fair peer-review system, which is all easy to use. Visit http://www. dovepress.com/testimonials.php to read real quotes from published authors. 